\title{
Thermal stability of simple tetragonal and hexagonal diamond germanium
}

L. Q. Huston, B. C. Johnson, B. Haberl, S. Wong, J. S. Williams, and J. E. Bradby

Citation: Journal of Applied Physics 122, 175108 (2017); doi: 10.1063/1.5002705

View online: https://doi.org/10.1063/1.5002705

View Table of Contents: http://aip.scitation.org/toc/jap/122/17

Published by the American Institute of Physics

\section{Articles you may be interested in}

Thermoelectric band engineering: The role of carrier scattering

Journal of Applied Physics 122, 175102 (2017); 10.1063/1.4994696

Cold nanoindentation of germanium

Applied Physics Letters 111, 021901 (2017); 10.1063/1.4993163

Surface enhanced Raman spectroscopic substrate utilizing gold nanoparticles on carbon nanotubes Journal of Applied Physics 122, 175107 (2017); 10.1063/1.4999333

Ballistic thermoelectric properties of nitrogenated holey graphene nanostructures Journal of Applied Physics 122, 174302 (2017); 10.1063/1.4999497

Three-dimensional reconstruction of neutron, gamma-ray, and x-ray sources using spherical harmonic decomposition

Journal of Applied Physics 122, 175901 (2017); 10.1063/1.4986652

Parametric design criteria of an updated thermoradiative cell operating at optimal states Journal of Applied Physics 122, 174505 (2017); 10.1063/1.4998002

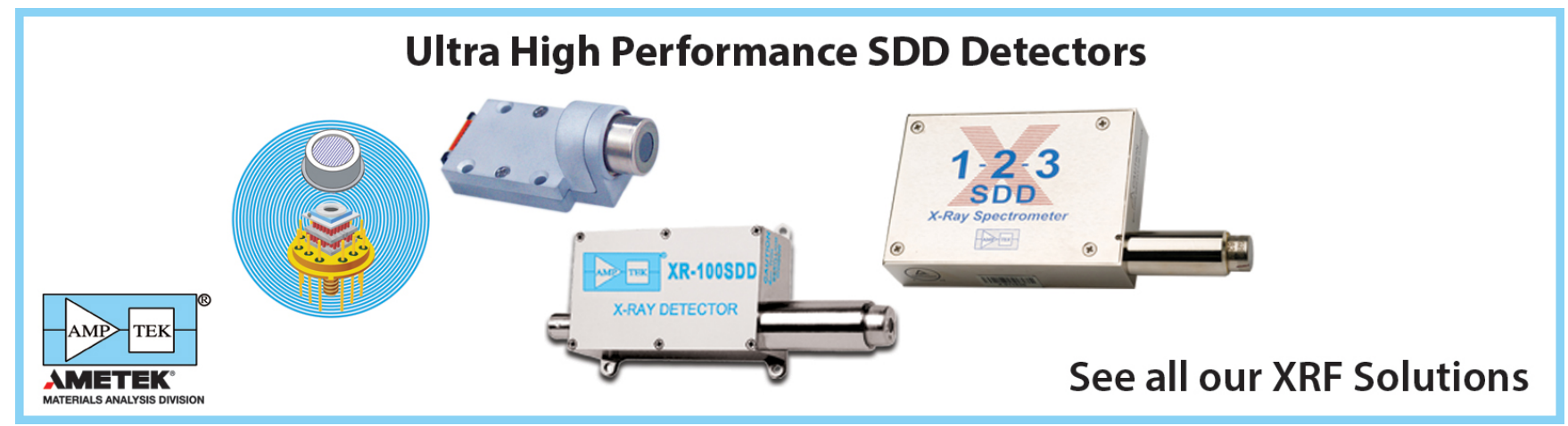




\title{
Thermal stability of simple tetragonal and hexagonal diamond germanium
}

\author{
L. Q. Huston, ${ }^{1, a)}$ B. C. Johnson, ${ }^{2}$ B. Haberl, ${ }^{3}$ S. Wong, ${ }^{1, b)}$ J. S. Williams, ${ }^{1}$ and J. E. Bradby ${ }^{1}$ \\ ${ }^{1}$ Department of Electronic Materials Engineering, Research School of Physics and Engineering, \\ The Australian National University, Acton, ACT 2601, Australia \\ ${ }^{2}$ School of Physics, University of Melbourne, Victoria 3010, Australia \\ ${ }^{3}$ Neutron Scattering Division, Neutron Sciences Directorate, Oak Ridge National Laboratory, Oak Ridge, \\ Tennessee 37831, USA
}

(Received 31 August 2017; accepted 21 October 2017; published online 7 November 2017)

\begin{abstract}
Exotic phases of germanium, that form under high pressure but persist under ambient conditions, are of technological interest due to their unique optical and electrical properties. The thermal evolution and stability of two of these exotic Ge phases, the simple tetragonal (st12) and hexagonal diamond (hd) phases, are investigated in detail. These metastable phases, formed by high pressure decompression in either a diamond anvil cell or by nanoindentation, are annealed at temperatures ranging from 280 to $320^{\circ} \mathrm{C}$ for st12-Ge and 200 to $550^{\circ} \mathrm{C}$ for hd-Ge. In both cases, the exotic phases originated from entirely pure Ge precursor materials. Raman microspectroscopy is used to monitor the phase changes ex situ following annealing. Our results show that hd-Ge synthesized via a pure form of a-Ge first undergoes a subtle change in structure and then an irreversible phase transformation to dc-Ge with an activation energy of $(4.3 \pm 0.2) \mathrm{eV}$ at higher temperatures. St12-Ge was found to transform to dc-Ge with an activation energy of $(1.44 \pm 0.08) \mathrm{eV}$. Taken together with results from previous studies, this study allows for intriguing comparisons with silicon and suggests promising technological applications. Published by AIP Publishing. https://doi.org/10.1063/1.5002705
\end{abstract}

\section{INTRODUCTION}

It is well established that when germanium $(\mathrm{Ge})$, both in its amorphous (a-Ge) and diamond cubic (dc-Ge, $F d \overline{3} m)$ form, is placed under $\sim 10 \mathrm{GPa}$ of pressure, a phase transformation to the metallic $\beta$-Sn structure ( $\beta$-Sn-Ge, I4 $1 /$ amd $)$ can occur. ${ }^{1-3}$ This semiconductor-metal transition is accompanied by a $\sim 20 \%$ volume collapse causing the $\beta$-Sn-Ge to become nanocrystalline. Upon pressure release, this phase does not transform back into dc-Ge at room temperature. Instead, a number of nanocrystalline metastable phases with a density significantly higher than that of dc-Ge form. ${ }^{2,4,5}$ These phases include a simple tetragonal phase (st12-Ge, $P 4_{3} 2_{1} 2$ ) (Refs. 2 and 4) and a body centered cubic structure (bc8-Ge, Iâ3). ${ }^{5}$ One parameter that can drive the transition to one phase over the other is temperature, with room temperature decompression resulting in st12-Ge and temperatures below the dry ice temperature resulting in bc8-Ge. ${ }^{5-7} \mathrm{~A}$ further mechanism that can determine the final phase is the rate of decompression, where slow unloading yields st12-Ge and fast unloading results in bc8-Ge. ${ }^{8}$ More recently, it was found that shear is also a critical factor in determining the final phase. A quasi-hydrostatic environment always results in the formation of a rhombohedral phase ( $\mathrm{r} 8-\mathrm{Ge}, R \overline{3})$ followed by bc8-Ge upon room-temperature unloading independent of the unloading rate. ${ }^{9}$ When left at ambient pressure and temperature, this bc8-Ge is kinetically unstable and transforms to the hexagonal diamond phase (hd-Ge, $\left.P 6_{3} / m m c\right)$ over a few days. ${ }^{6,8,10,11}$ In contrast, a high shear environment on decompression produces st12-Ge. ${ }^{9}$ This is

\footnotetext{
a) larissa.huston@anu.edu.au.

${ }^{b)}$ Current address: School of Science, RMIT University, GPO Box 2476V, Melbourne, VIC 3000, Australia.
}

consistent with a high pressure torsion study at ambient that produced a mixture of st12-Ge and dc-Ge. ${ }^{12}$ Different from bc8-Ge, st12-Ge has been reported to be kinetically stable at ambient conditions. ${ }^{2}$ For consistency with literature, the phases will be referred to by their abbreviated names for the remainder of the paper (e.g. dc-Ge).

Both st12-Ge and hd-Ge have been predicted to have properties of technological importance. The hd-Ge structure has been predicted to be a narrow $(0.55 \mathrm{eV})$ direct bandgap semiconductor. ${ }^{13,14}$ Comparisons with modelling performed for st12-Si suggest that st-12-Ge may act as a superconductor at low temperatures when suitably doped. ${ }^{13}$ In addition, a recent study of the properties of st12-Ge found that it has an indirect bandgap of $0.59 \mathrm{eV}$ and a direct optical transition at $0.74 \mathrm{eV} .{ }^{15}$ Furthermore, if these exotic Ge structures could be synthesized in the form of hydrogenated nanoparticles, they could be highly advantageous for solar power conversion. ${ }^{16}$ All these properties provide a strong motivation for further investigation of these exotic phases. However, to utilize their promising electrical properties it is vital to understand their thermal evolution, since incorporating such phases into devices requires the materials to be able to remain functional throughout several high temperature processing steps. Indeed, previous reports the stability of st12-Ge have shown that it anneals to dc-Ge within hours at $200{ }^{\circ} \mathrm{C}$ (Ref. 1) and within minutes at $245-285^{\circ} \mathrm{C}$, with an activation energy of $\sim 160 \mathrm{~kJ} /$ mol or $\sim 1.66 \mathrm{eV} .^{6} \mathrm{~A}$ more recent study found a transition temperature near $207^{\circ} \mathrm{C} .{ }^{15}$ The dc-Ge formed after annealing st $12-\mathrm{Ge}$ at $300^{\circ} \mathrm{C}$ was found to be nanocrystalline and contain stacking faults and nanotwins. ${ }^{17}$ In contrast, hd-Ge was found to transform to dc-Ge in the $485-550{ }^{\circ} \mathrm{C}$ regime..$^{6,18}$ One study found that this transition was reversible, attributing this reversibility to the critical interplay of impurities and 
defects. ${ }^{6}$ This current work expands on these previous studies and presents a detailed study of the thermal stability and evolution of st12-Ge and hd-Ge. Here, we synthesized the exotic phases from an entirely pure Ge precursor material, thus removing any ambiguities about defect and impurity contributions. In the case of st12-Ge, semiconductor grade $\mathrm{Cz} \mathrm{Ge}$ powdered prior to loading was used, while self-ion-implanted a-Ge created directly from the wafer was used to create hd-Ge. The annealing behavior of both these phases was examined over a large range of temperatures and times to extract the activation energy of the transition to dc-Ge. In the case of hd-Ge, care was taken to remove all disorders in its starting structure by using a pre-annealed hd-Ge for the activation study. This study also provides insight into the phase behavior of exotic silicon phases, which are potentially important to future semiconductor applications.

\section{EXPERIMENTAL SECTION}

\section{A. St12-Ge}

The metastable Ge phases investigated in this work were prepared using both a diamond-anvil cell (DAC) and nanoindentation under conditions which have previously been determined to reliably produce the st12 (Ref. 9) and hd phases (Ref. 19), respectively. The st12-Ge samples were synthesized in a DAC with a $400 \mu \mathrm{m}$ culet size using dc-Ge powder, which was formed by grinding a $\mathrm{Cz}$ n-type $(100 \mathrm{dc}-\mathrm{Ge})$ wafer in an agate mortar and pestle as a starting material. The resulting powder was loaded into a $\sim 50 \mu \mathrm{m}$ deep $\sim 200 \mu \mathrm{m}$ hole in a steel gasket. No pressure medium was used in order to induce the maximum shear possible in the sample. The samples were loaded until it was clear that all the dc-Ge had been transformed to the metallic $(\beta-\mathrm{Sn})$ phase as confirmed by changes in its appearance to a light grey color which indicated the presence of a metallic phase. The samples were then unloaded to ambient pressure with unloading times ranging between 10 and $60 \mathrm{~s}$. The gasket containing the sample was removed from the DAC and placed in a Linkam stage using silver paint to ensure good thermal contact. An InVia Renishaw Raman spectrometer equipped with a $532 \mathrm{~nm}$ laser, a $50 \times$ lens, and a $24001 / \mathrm{mm}$ grating was used to perform Raman microspectroscopy at two locations on the sample before and after annealing. All Raman spectra were taken at $20^{\circ} \mathrm{C}$ for $30 \mathrm{~s}$ and at a laser power of $\sim 2 \mathrm{~mW}$ so that the sample did not undergo any unintended phase transformations induced by the laser. This laser power did not alter the structure of the sample, as confirmed by taking the Raman spectra of an unannealed indent starting at lower laser powers and gradually increasing the laser power until the spectrum changed. Thus, the laser power chosen was the higher power available that did not change the Raman signal. The samples were annealed with the Linkam stage with flowing $\mathrm{N}_{2}$ gas for 3 to $20 \mathrm{~min}$ (depending on the temperature and previous annealing times) and then cooled to room temperature in approximately $100-200 \mathrm{~s}$ before each measurement. The Linkam stage was chosen for the DAC prepared samples to avoid contamination of a furnace tube with iron from the gasket. The sample was again heated, cooled, and measured. This was repeated until no change in the final Raman spectrum was observed. The following annealing temperatures for st12-Ge were employed: $280,290,300$, and $320^{\circ} \mathrm{C}$. A new sample was used for each annealing temperature.

\section{B. $\mathrm{Hd}-\mathrm{Ge}$}

The hd-Ge structure was prepared via nanoindentation using ion-implanted a-Ge as the starting material, following the conditions of a previous study. ${ }^{19}$ The implanted layer thickness was found to be $1800 \mathrm{~nm}$ using transmission electron microscopy (TEM). A $20 \mu \mathrm{m}$ radius spherical diamond tip was used to indent the a-Ge to a maximum load of $700 \mathrm{mN}$ in an Ultra-Micro-Indentation-System 2000, resulting in an indentation depth of $1200 \mathrm{~nm}$ as observed in Ref. 19. These conditions were previously shown to reliably result in the formation of phase transformed regions of $\mathrm{r} 8$ / bc8-Ge and hd-Ge that were $\sim 11 \mu \mathrm{m}$ in diameter and extended down to $\sim 1.3 \mu \mathrm{m}$ from the surface. ${ }^{10,11,19}$ After indentation, the samples were left for at least two weeks at ambient temperature such that the $\mathrm{bc} 8 / \mathrm{r} 8$-Ge transformed fully to hd-Ge. ${ }^{9}$ A Raman spectrum was recorded from each individual indent using a $532 \mathrm{~nm}$ laser with a $100 \times$ objective, a laser power of $\sim 0.5 \mathrm{~mW}$, and a $2400 \mathrm{l} / \mathrm{mm}$ grating for $30 \mathrm{~s}$. It should be noted that the laser spot size and penetration depth were significantly smaller than the phase transformed region. These samples were then annealed in a tube furnace in a $100 \mathrm{ccm}$ flowing $\mathrm{N}_{2}$ atmosphere with increasing time durations until no further change was observed in the Raman spectra. After each anneal, a Raman spectrum for each indent was recorded under the same conditions. There were two annealing temperature ranges used in the hd-Ge experiment: the lower temperature range $(200,215,230$, 250 , and $300^{\circ} \mathrm{C}$ ) and the higher temperature range (500, 515,530 , and $550^{\circ} \mathrm{C}$ ). The annealing times ranged from $5 \mathrm{~min}$ to $\sim 1000 \mathrm{~min}$. For the higher temperature range, the samples were first annealed for $10 \mathrm{~min}$ at $500^{\circ} \mathrm{C}$ to induce a structural change, as discussed later, which is a precursor to annealing at higher temperatures. The accumulation times for the lower and higher temperature ranges were $30 \mathrm{~s}$ and $90 \mathrm{~s}$, respectively.

Transmission electron microscopy (TEM) of the hd-Ge samples was performed using focused ion beam milling (FEI Helios Nanolab) and the ex situ plucking method. ${ }^{20}$ Platinum was initially deposited to protect the surface before the cross-section was cut through the residual indent. The final milling was done at $16 \mathrm{keV}$. TEM images were taken using a Phillips CM300 and a Jeol $2100 \mathrm{~F}$ instrument at $300 \mathrm{kV}$ and $200 \mathrm{kV}$, respectively. The effective size of the selected area apertures ranged from $240 \mathrm{~nm}$ to $640 \mathrm{~nm}$. Care was taken to ensure that the apertures were positioned such that the diffraction pattern was only taken from the phase transformation zone.

\section{GENERAL ANNEALING BEHAVIOR}

\section{A. St12-Ge}

Figure 1 shows the representative behavior of st12-Ge upon thermal annealing. In this example, annealing to $280^{\circ} \mathrm{C}$ for up to $75 \mathrm{~min}$ is displayed. Note that the thermal evolution 


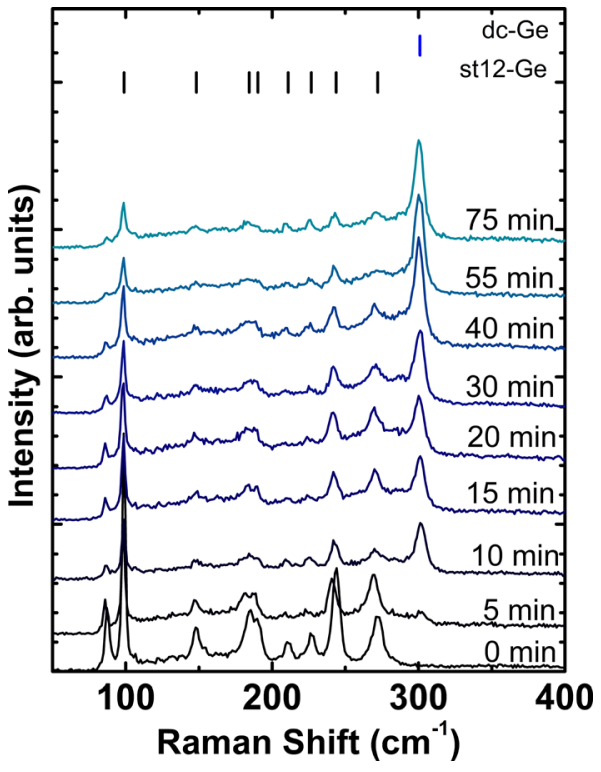

FIG. 1. Raman spectra from a st12-Ge sample formed in a DAC and annealed up to $75 \mathrm{~min}$ at $280^{\circ} \mathrm{C}$. The marks at the top indicate the calculated positions of the Raman peaks of st12-Ge and dc-Ge. ${ }^{21}$

at other temperatures was comparable and is not shown here. The spectrum of near-pure st12-Ge before annealing is shown at the bottom of the plot in black. This spectrum contains eight clear peaks at 87, 99, 148, 184, 190, 211, 226, 243, and $271 \mathrm{~cm}^{-1}$. These peaks have an excellent agreement with those previously calculated for st12-Ge. ${ }^{21}$ After a $5 \mathrm{~min}$ anneal, the transverse optical (TO) Raman band at $301 \mathrm{~cm}^{-1}$ of the dc-Ge phase starts to form while the st12-Ge peaks decrease in intensity. After $75 \mathrm{~min}$ of annealing at $280^{\circ} \mathrm{C}$, the Raman spectrum consists predominantly of dc-Ge with only a small amount of st12-Ge remaining. It should be noted that no peaks other than the st12-Ge peaks and the dc-Ge peak were observed. Therefore, it is clear that st12-Ge transforms directly into dc-Ge, consistent with previous studies. ${ }^{2,7,15}$ As expected, at higher temperatures this transformation occurs at a faster rate. For example, at $300^{\circ} \mathrm{C}$, st12-Ge completely anneals to dc-Ge within $5 \mathrm{~min}$.

\section{B. Hd-Ge}

Figure 2(a) shows the Raman spectra of indents containing hd-Ge before and after annealing at $200^{\circ} \mathrm{C}$ for $250 \mathrm{~min}$. The spectra from dc-Ge and the pristine a-Ge formed by ionimplantation are shown for reference. The hd-Ge spectrum is composed of two distinct bands. The first is a broad band at $\sim 76 \mathrm{~cm}^{-1}$ that corresponds to the transverse acoustic mode of the amorphous or disordered material. This broad band remains after annealing but decreases in its relative height, which is consistent with a-Ge not transforming into dc-Ge until $\sim 460{ }^{\circ} \mathrm{C}$. $^{22} \mathrm{~A}$ second broad peak centered at around $295 \mathrm{~cm}^{-1}$ is also observed in the spectra from the indent (before annealing). This peak has been previously studied and shown to be a complex combination of three Raman bands: a broad a-Ge peak (as seen in the a-Ge spectrum) at $270 \mathrm{~cm}^{-1}$ and two sharper hd-Ge bands at (1) $290 \mathrm{~cm}^{-1}$ and (2) $\sim 300 \mathrm{~cm}^{-1}$. ${ }^{11}$ The fits for the three hd-Ge spectra are shown

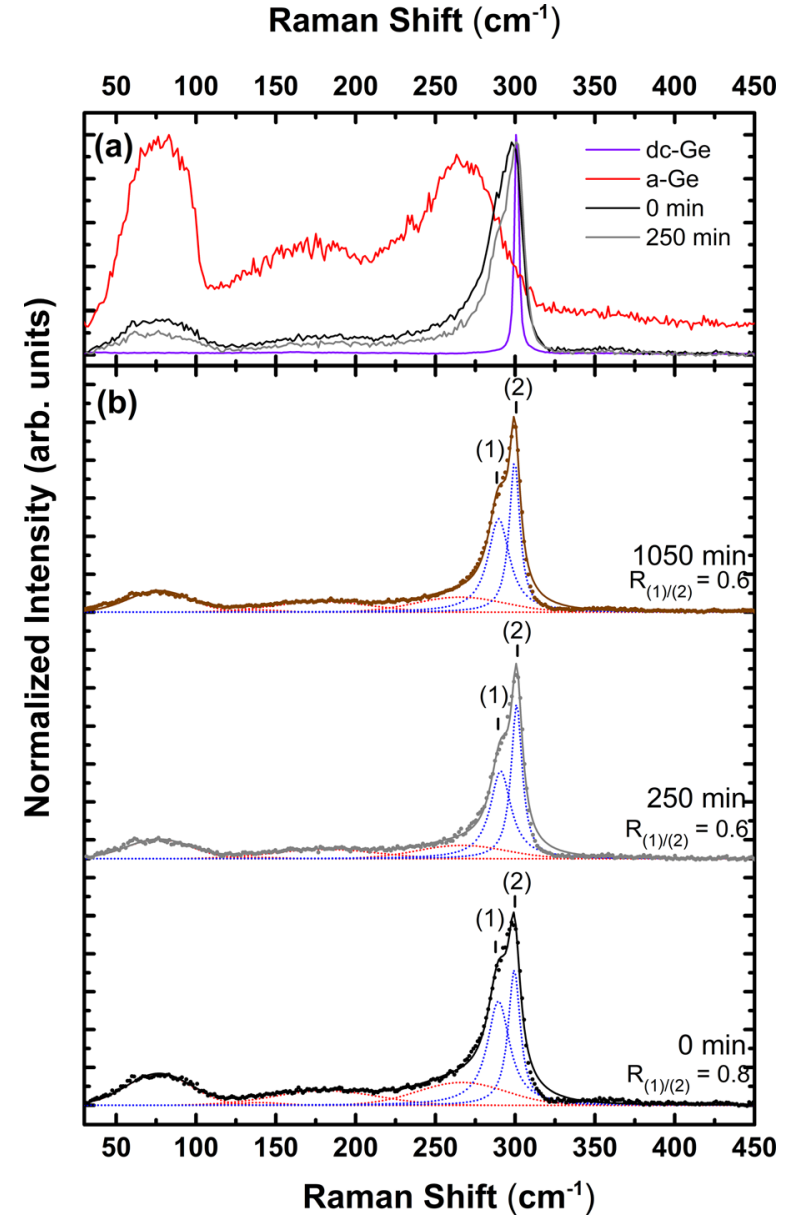

FIG. 2. (a) The Raman spectrum of an indent before annealing and after annealing at $200^{\circ} \mathrm{C}$ for $250 \mathrm{~min}$. The spectra of dc-Ge and a-Ge are shown for reference. (b) The curve fits and their components for the hd-Ge spectra, which have been annealed for 0,250 , and $1050 \mathrm{~min} . \mathrm{R}_{(1) /(2)}$ is the ratio of the heights of the fitted peaks labeled (1) and (2).

in Fig. 2(b). It should be noted that the peak at $300 \mathrm{~cm}^{-1}$ is neither from dc-Ge nor from the a-Ge substrate below, as confirmed with TEM and discussed further below. Interestingly, from these fits, we see a change in the relative intensity of the two characteristic hd-Ge peaks at 290 and $300 \mathrm{~cm}^{-1}$. Initially, the ratio of intensity of the two peaks $\left(R_{(1) /(2)}\right)$ is 0.8 but after $250 \mathrm{~min}$, the $290 \mathrm{~cm}^{-1}$ peak is lower relative to the $300 \mathrm{~cm}^{-1}$ peak, with $\left(R_{(1) /(2)}\right)=0.6$, while after $1050 \mathrm{~min}$, there is no further change in $\left(R_{(1) /(2)}\right)$. We note that this relative change in intensity indicates a subtle change in the structure. It is also worth noting that the hd-Ge signal becomes stronger relative to the a-Ge signal. These changes are presumably similar to the mechanism reported in $\mathrm{Si}$, where bc8-Si undergoes a two step transition to hd-Si where it first turns into to a mixture of disordered and microcrystalline form of hd-Si before ordering into hd-Si. ${ }^{23}$ This change is now investigated further using transmission electron microscopy (TEM) performed ex situ after annealing at this temperature.

Selected area diffraction patterns (SADPs) of one indentation impression that was not annealed and of three indentation impressions after annealing at different conditions are shown in Fig. 3. For the SADP of the sample that was not annealed, the majority of the reflections were indexed to 

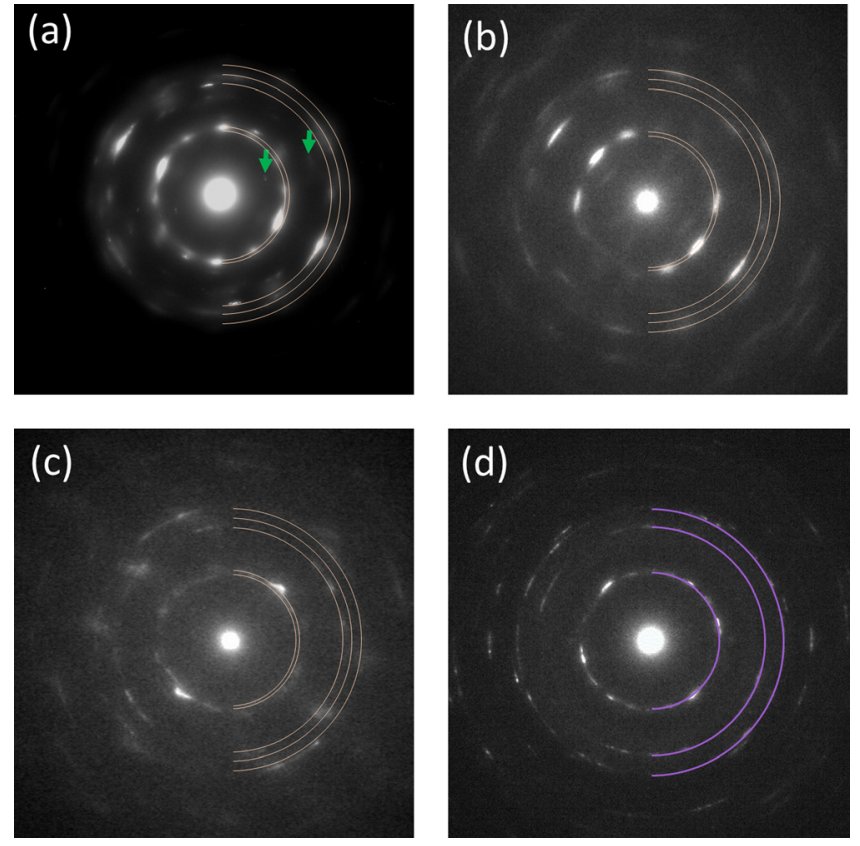

FIG. 3. Selected area diffraction patterns taken from the transformed region underneath an indent impression for (a) no annealing and indentation impressions, which have been annealed to (b) $250{ }^{\circ} \mathrm{C}$ for $250 \mathrm{~min}$, (c) $500^{\circ} \mathrm{C}$ for $10 \mathrm{~min}$, and (d) $515^{\circ} \mathrm{C}$ for $120 \mathrm{~min}$. The arrows indicate the reflections corresponding to bc8-Ge, and the light orange and purple circles correspond to hd-Ge and dc-Ge, respectively.

hd-Ge, consistent with the Raman spectrum. Additionally, however, a faint reflection, indicated by arrows) is also found at $4.5 \AA$ as also reported by Williams et al. ${ }^{11}$ This faint reflection could be attributed to (110) bc 8 or (112) st12 with lattice parameters of $\mathrm{a}=6.36 \AA$ and $\mathrm{a}=5.92 \AA, \mathrm{c}=6.98 \AA$, respectively, at ambient pressures. ${ }^{5}$ However, this one faint reflection is not sufficient to unambiguously index a full unit cell. Also, no evidence of st12-Ge has been observed in any Raman study on such phase transitions induced in a-Ge, and only bc8-Ge, r8-Ge, and hd-Ge have been observed. Furthermore, bc8-Ge is clearly a transient phase along this hydrostatic transition pathway towards hd-Ge. We thus suggest that a small remnant of bc8/r8-Ge is observed in these residual indent impressions even after some 'aging' and FIB processing. This is consistent with a previous study that reported full elimination of bc8-Ge on the way to hd-Ge at $100{ }^{\circ} \mathrm{C}$ only. ${ }^{6}$

An SADP taken from the phase transformed region of an indent which was annealed in the lower annealing temperature range, at $250{ }^{\circ} \mathrm{C}$ for $250 \mathrm{~min}$, is shown in Fig. 3(b). Here, all the reflections can be indexed to hd-Ge and there is no longer evidence of the reflection seen at $\sim 4.5 \AA$. Additionally, the reflections appear to be sharper with less diffuse scattering around them. Clearly based on the SADP analysis, no evidence of dc-Ge is observed in the phase transformed region. Thus, hd-Ge does not transform to dc-Ge in this lower annealing temperature range. Instead, we suggest that the observed changes in the Raman spectra of hd-Ge in this lower temperature regime are due to a combination of annealing of a disordered form of hd-Ge to a more ordered one that is similar to the annealing behavior of hd-Si (Ref. 23) and the full conversion of all remaining bc $8 / \mathrm{r} 8-\mathrm{Ge}$ to hd-Ge. It appears that this conversion does not fully take place at room temperature. For the study of the kinetics of more ordered hd-Ge, it is thus clearly desirable to choose a 'starting point' from which only the hd-Ge to dc-Ge transition occurs as carried out in this current study. Figures 3(c) and 3(d) show the SADPs of indents annealed at higher temperatures. The SADP in Fig. 3(c) is from an indent that was annealed at $500^{\circ} \mathrm{C}$ for $10 \mathrm{~min}$. The reflections in this SADP show that hd-Ge is still present, however some dc-Ge may be present due to the presence of reflections with a sharper radial distribution at d-spacings that dc-Ge and hd-Ge have in common. It is worth noting that the amorphous substrate (not shown) was found to have crystallized into dc-Ge, consistent with the temperature dependence of solid phase epitaxial growth of a-Ge. ${ }^{18}$ Finally, Fig. 3(d) shows an SADP for an indent which has been annealed for $120 \mathrm{~min}$ at $515^{\circ} \mathrm{C}$. In this SADP, there is clear evidence of dc-Ge due to the presence of less rings but more sharp reflections spread throughout the ring. The Raman spectra of similar indents show the presence of hd-Ge which cannot be ruled out from the SADP due to many common reflections between the two phases.

From the TEM results, it is clear that hd-Ge transforms directly to dc-Ge at temperatures above $500{ }^{\circ} \mathrm{C}$. To further understand this process, we turn to Raman spectroscopy. Figure 4 shows the Raman spectra of the thermal evolution when hd-Ge is annealed at $515^{\circ} \mathrm{C}$. The initial spectrum is taken after annealing the sample for $10 \mathrm{~min}$ at $500^{\circ} \mathrm{C}$ as previously discussed. The interpretation of the Raman data is not straight-forward due to the hd-Ge modes (290, 301, and $302 \mathrm{~cm}^{-1}$ ) overlapping with the dc-Ge mode at $301 \mathrm{~cm}^{-1}$, making the de-convolution of the spectra challenging. To address this, we have focused our analysis primarily on the band at $290 \mathrm{~cm}^{-1}$. After annealing, this $290 \mathrm{~cm}^{-1}$ band appears as a shoulder and decreases in relative intensity up to the maximum annealing time of $400 \mathrm{~min}$ after which most of the hd-Ge is transformed to dc-Ge. This decrease is shown more clearly in the inset. The equivalent change in the relative intensity at $550^{\circ} \mathrm{C}$ occurs much earlier at $30 \mathrm{~min}$. We

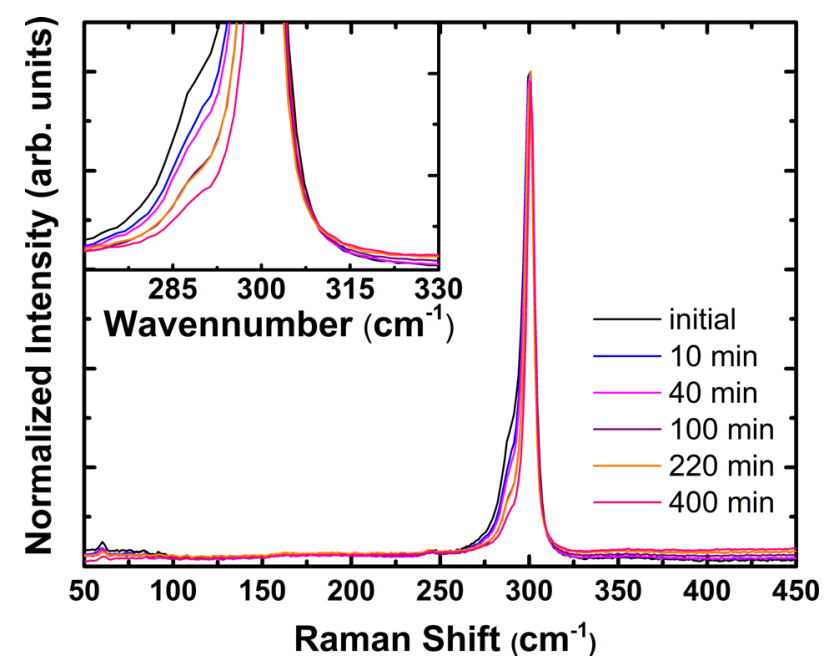

FIG. 4. The change in the normalized Raman spectrum of hd-Ge formed by the nanoindentation annealed at $515^{\circ} \mathrm{C}$. The inset shows an expanded view of the intensity. Raman spectra are normalized by dividing by the maximum intensity between 275 and $320 \mathrm{~cm}^{-1}$. 
note that there is no evidence that the hd to dc transformation is reversible as previously suggested by Brazhkin et al., ${ }^{6}$ possibly due to the fact that pure precursor materials were used in the current study.

\section{ACTIVATION ENERGY}

The full set of temperature and time data from both the st12 and hd-Ge annealing processes was analyzed to calculate the respective activation energies. This was done by measuring the relative Raman peak heights obtained through multiLorentzian fits to each spectrum. The spectra annealed at each temperature and time were used to determine the characteristic transformation rate, $\mathrm{k}$. This rate was defined as the inverse of the time it takes for the peak intensity to reduce to $1 / \mathrm{e}$ of its starting intensity (in $\mathrm{s}^{-1}$ ). The method used to determine $\mathrm{k}$ took account of differences in the absolute Raman (peak) intensities for the different phases. It was assumed that, within the laser excitation volume, the phases are homogeneously distributed throughout the annealing sequence.

The following Arrhenius equation was used to fit the data and determine the activation energy of the phase transformation:

$$
\mathrm{k}=\mathrm{Ce}^{-\frac{\mathrm{E}_{\mathrm{a}}}{\mathrm{k}_{\mathrm{b}} \mathrm{T}}}
$$

where $\mathrm{E}_{\mathrm{a}}$ is the activation energy in $\mathrm{eV}, \mathrm{C}$ is a constant, $\mathrm{T}$ is the temperature in $\mathrm{K}$, and $\mathrm{k}_{\mathrm{b}}$ is the Boltzmann's constant. Figure 5 shows the relationship between $\ln (\mathrm{k})$ and the annealing temperature in an Arrhenius plot. The transformation of both phases is found to be linear within error and can therefore be described by single activation energy. From these fits, the thermally activated st12-Ge to dc-Ge transformation is found to be characterized by $\mathrm{E}_{\mathrm{a}}=(1.44 \pm 0.08) \mathrm{eV}$ and is in

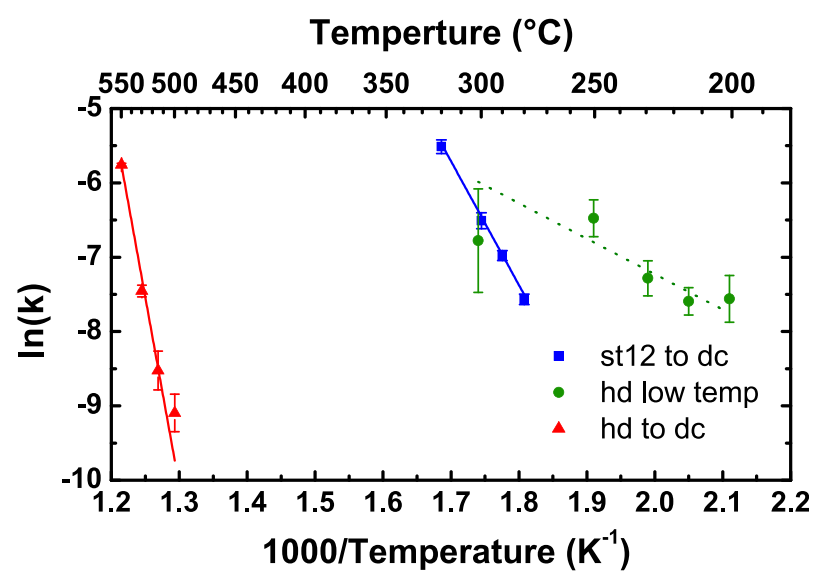

FIG. 5. Arrhenius plot of the st12-Ge to dc-Ge phase transition and the hd-Ge to dc-Ge transition, where $\mathrm{k}=$ time in $\mathrm{s}^{-1}$ for the hd-Ge or st12-Ge Raman peak to reduce to $1 / \mathrm{e}$ of the initial intensity. The error represented on the graph comes from uncertainty in the fit from the peak heights to obtain $\mathrm{k}$. It is larger for hd-Ge due to the dc-Ge and hd-Ge peaks overlapping. The linear fits give activation energies of $1.44 \pm 0.08 \mathrm{eV}$ and $4.3 \pm 0.2 \mathrm{eV}$ for these transitions, respectively. $\ln (\mathrm{C})$ is $22.6 \pm 1.6$ and $56 \pm 2$ for the st $12-\mathrm{Ge}$ to dc-Ge and hd-Ge to dc-Ge transformations, respectively. The dotted green line represents the low temperature behavior of hd-Ge, where an effective composite activation energy of $(0.5 \pm 0.1) \mathrm{eV}$ was extracted. This is possibly due to the full conversion of bc8/r8-Ge to hd-Ge and also some ordering and defect annihilation within grain boundaries of hd-Ge. good agreement with a previous study. ${ }^{10}$ The hd-Ge to dc-Ge transition has a higher activation energy of $\mathrm{E}_{\mathrm{a}}=(4.3 \pm 0.2)$ $\mathrm{eV} . \ln (\mathrm{C})$ is $22.6 \pm 1.6$ and $56 \pm 2$ for the st12-Ge to dc-Ge and hd-Ge to dc-Ge transformations, respectively. The error arises from the uncertainty in the fits. These trends show that the hd-Ge is much more thermally stable than st12-Ge and a-Ge.

A similar method was used for the change of the hd-Ge peaks observed between 200 and $300{ }^{\circ} \mathrm{C}$ and an activation energy of $(0.5 \pm 0.1) \mathrm{eV}$ is obtained. This change is probably an effective composite activation energy involving the full conversion of bc8/r8-Ge to hd-Ge and also some ordering and defect annihilation within grain boundaries of hd-Ge.

\section{DISCUSSION}

To use these results to gain further insight into the stability of metastable phases, it is better to compare the behavior of Ge to that of its 'sister' group 14 element, Si. Like dc-Ge, dc-Si undergoes a phase transformation to $(\beta-\mathrm{Sn})-\mathrm{Si}$ above $10 \mathrm{GPa}^{1,2}$ Upon unloading in a DAC, $(\beta$-Sn $)-\mathrm{Si}$ transforms equally to various metastable phases rather than dc-Si. Initially, r8-Si is formed upon decompression to $\sim 9 \mathrm{GPa}^{24}$ This transforms further to bc8-Si upon unloading to $3 \mathrm{GPa}^{24}$ The bc8-Si in turn anneals to hd-Si upon heating to around $200^{\circ} \mathrm{C}$ (Ref. 25) and hd-Si transforms to dc-Si in the $720-775^{\circ} \mathrm{C}$ regime when made in a high pressure cell (Ref. 6). St12-Si has not been made using conventional pressure methods. However, small amounts of this phase have been obtained by laser induced microexplosions but not in sufficient quantities to determine its thermal stability behavior. ${ }^{26}$ Figure 6 summarizes the transformation data from the present work and for other metastable Ge phases [a-Ge (Ref. 27) and r8-Ge (Ref. 10)]. Based on the literature values, a-Ge crystallizes to dc-Ge at around $450{ }^{\circ} \mathrm{C}$ with an activation energy of $2.15 \mathrm{eV}$ (Ref. 27), while the a-Si to dc-Si transition occurs at above $650{ }^{\circ} \mathrm{C}$ with an activation energy of $2.70 \mathrm{eV} .^{28}$ Thus, all the Si phase transformations occur at higher temperatures than their equivalent transformations in Ge. This might be expected given the different respective melting points of these materials, which are indicative of the relative stability and bond strengths. Indeed,

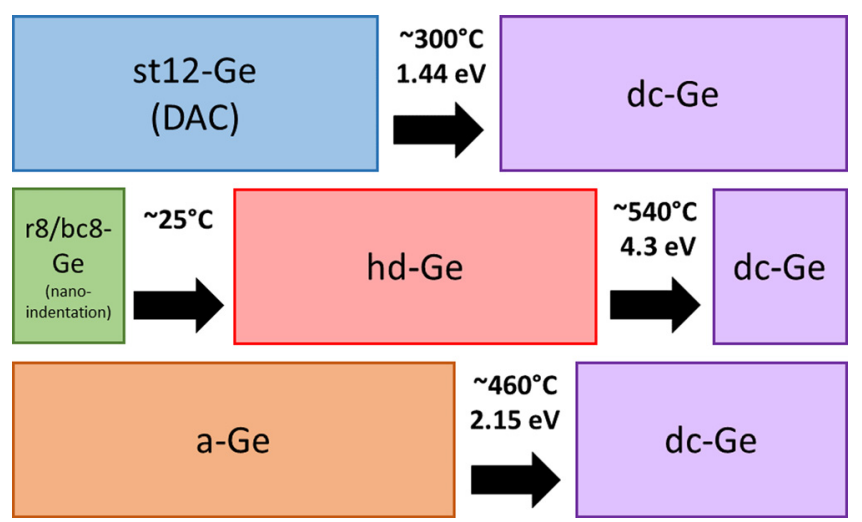

FIG. 6. Summary of the known transformations between various metastable phases of Ge upon heating. The arrows indicate a thermally induced phase transformation. The a-Ge to dc-Ge transition activation energy and the r8-Ge to hd-Ge transition temperature are taken from Refs. 27 and 11, respectively. 
assessment of the transformation temperatures $\left(\mathrm{T}_{\text {Transf }}\right)$ relative to the $\mathrm{Si}$ and Ge melting temperatures $\left(\mathrm{T}_{\mathrm{m}}\right)$ demonstrates this strong correlation. The various $\mathrm{T}_{\mathrm{c}}, \mathrm{T}_{\mathrm{m}}$, and corresponding ratios are summarized in Table $\mathrm{I}$.

Clearly, the scaling of $\mathrm{T}_{\text {transf }}$ to $\mathrm{T}_{\mathrm{m}}$ demonstrates an excellent agreement between the kinetic stability of the various exotic phases of $\mathrm{Ge}$ and $\mathrm{Si}$. Based on these extremely similar ratios for all transitions, a $\mathrm{T}_{\text {Transf }} / \mathrm{T}_{\mathrm{m}}$ ratio of 0.47 can also be postulated for st12-Si. This corresponds then to a $\mathrm{T}_{\text {Transf }}=\sim 520^{\circ} \mathrm{C}$ for the st $12-\mathrm{Si}$ to dc-Si transition. This insight shows that the st12-Si phase may be potentially useful once it can be formed selectively.

Returning to Ge, we will now comment on the technical implications of this study for the possible use of the metastable Ge phases in device applications. Provided a pre-anneal is performed to complete the bc8-Ge to hd-Ge conversion, the kinetic stability of hd-Ge means that it will not be affected by low temperature processing, which involves annealing at $400{ }^{\circ} \mathrm{C}^{29}$ However, the a-Ge substrate will undergo some solid phase epitaxial regrowth at this temperature (Ref. 27); thus, it will be important to understand the electrical properties of the hd-Ge/dc-Ge and hd-Ge/a-Ge interfaces as these will have implications on any device produced from the indents of hd-Ge. It should also be mentioned that the annealing behavior of this phase in the lower temperature range may slightly alter the electrical properties during any low temperature processing. Hence, any change in the electrical properties before and after low temperature treatment should also be studied. As for st12-Ge, the kinetic stability of the st12 phase suggests that this structure will be able to withstand solar radiation without transforming to dc-Ge. Moreover, as st12-Si was predicted to be superconducting, ${ }^{13}$ there is a strong possibility that st12-Ge is also a superconductor. Finally, it is interesting to consider that understanding these forms of Ge may be useful for other related systems. For example, testing the hardness of hd-Ge could give insight into the relative hardness of the hd and dc phase of $\mathrm{C}$, since the hexagonal form of $\mathrm{C}$

TABLE I. The transformation temperatures $\left(\mathrm{T}_{\mathrm{Transf}}\right)$ for melting $\left(\mathrm{T}_{\mathrm{m}}\right)$ and the transitions from amorphous, hd and st 12 to $\mathrm{dc}$, as well as from an r8/bc8 mixture to hd together with the corresponding $\mathrm{Tc} / \mathrm{Tm}$ ratios. The melting temperatures are taken from Ref. $31, \mathrm{~T}_{\text {Transf }}$ for the unseeded nucleation of the dc material from an amorphous precursor (Refs. 27 and 28), the various $\mathrm{T}_{\text {Transf }}$ of the exotic Ge phases from this work, $\mathrm{T}_{\text {Transf }}$ of hd-Si to dc-Si from (Ref. 6), and a $\mathrm{T}_{\mathrm{Transf}}$ of $150^{\circ} \mathrm{C}$ for a slightly pressurized mixture of bc8/r8Si from (Ref. 32) to replicate the similar residual stress state of the initial Ge indents on the verge of the bc 8 to $r 8$ transition.

\begin{tabular}{lcccc}
\hline \hline & $\begin{array}{c}T_{\text {Transf }} \text { Ge } \\
\left({ }^{\circ} \mathrm{C}\right)\end{array}$ & $\begin{array}{c}\mathrm{T}_{\text {Transf }} \mathrm{Si} \\
\left({ }^{\circ} \mathrm{C}\right)\end{array}$ & $\mathrm{T}_{\text {Transf }} / \mathrm{T}_{\mathrm{m}} \mathrm{Ge}^{\mathrm{a}}$ & $\mathrm{T}_{\text {Transf }} / \mathrm{T}_{\mathrm{m}} \mathrm{Si}^{\mathrm{a}}$ \\
\hline Melting $\mathrm{T}_{\mathrm{m}}$ & 940 & 1412 & 1 & 1 \\
Amorphous & $460^{\mathrm{b}}$ & $650^{\mathrm{b}}$ & 0.60 & 0.55 \\
hd & 500 & 740 & 0.64 & 0.60 \\
r8/bc8 & 25 & 150 & 0.25 & 0.25 \\
st12 & 300 & $520^{\mathrm{c}}$ & 0.47 & \\
\hline \hline
\end{tabular}

${ }^{\mathrm{a} C a l c u l a t e d ~ u s i n g ~ c o r r e s p o n d i n g ~ t e m p e r a t u r e s ~ i n ~} \mathrm{~K}$.

${ }^{b}$ Note that nucleation of the dc phase from a-Si or a-Ge is critically dependent on the exact properties of the amorphous film and thus a range of transformation temperature is more accurate.

${ }^{\mathrm{c}}$ Estimated based on $\mathrm{T}_{\text {Transf }} / \mathrm{T}_{\mathrm{m}}$ Ge and $\mathrm{T}_{\text {Transf }} / \mathrm{T}_{\mathrm{m}}$ Si being equal. (lonsdaleite) is predicted to have a hardness greater than that of diamond. ${ }^{30}$

\section{SUMMARY AND CONCLUSION}

In summary, the activation energies of st12-Ge to dc-Ge and hd-Ge to dc-Ge transitions have been measured. Pure precursor materials based on semiconductor grade $\mathrm{Ge}$ were used for both exotic phases. In the case of hd-Ge, a preanneal served to ensure good quality hd-Ge for the subsequent annealing study, since our results indicated that a lower temperature anneal is required to complete the conversion to hd-Ge. The Arrhenius dependence shows that the hdGe phase has greater kinetic stability than st12-Ge, allowing the possibility of low-temperature processing on this phase. Furthermore, the strong correlations between Si and Ge have been used to estimate the transition temperature of st12-Si to dc-Si to be around $525^{\circ} \mathrm{C}$, thus also making it capable of withstanding low temperature processing. This study thus clearly demonstrates that exotic phases of $\mathrm{Si}$ and $\mathrm{Ge}$ are sufficiently stable to be exploited for future semiconductor devices.

\section{ACKNOWLEDGMENTS}

The authors would like to thank AFAiiR (NCRIS), the Centre for Advanced Microscopy (CAM), and the Australian National Fabrication Facility (ANFF) for use of their facilities and technical support. This work was supported by the Australian Research Council under the Discovery Project Scheme. L.Q.H. is supported by an Australian Government Research Training Program Scholarship. J.E.B. acknowledges the ARC for the award of a Future Fellowship. B.H. was supported through a Weinberg Fellowship (ORNL) and the Neutron Scattering User Facilities (ORNL), supported by the U.S. Department of Energy, Office of Sciences, Basic Energy Sciences. The ORNL is funded under DOE-BES Contract No. DE-AC05-00OR22725 and the Alvin M. Weinberg Fellowship by the ORNL LDRD scheme under Project No. 7620.

${ }^{1}$ J. C. Jamieson, Science 139(3556), 762 (1963).

${ }^{2}$ F. P. Bundy and J. S. Kasper, Science 139(355), 340 (1963).

${ }^{3}$ S. Minomura and H. G. Drickamer, J. Phys. Chem. Solids 23(5), 451 (1962); S. Minomura, J. Phys. Colloques 42(NC4), 181 (1981).

${ }^{4}$ J. S. Kasper and S. M. Richards, Acta Cryst. 17(6), 752 (1964).

${ }^{5}$ C. H. Bates, F. Dachille, and R. Roy, Science 147(3660), 860 (1965).

${ }^{6}$ V. V. Brazhkin, A. G. Lyapin, S. V. Popova, and R. N. Voloshin, J. Exp. Theor. Phys. Lett. 56(3), 152 (1992), available at http://www.jetpletters.ac. ru/ps/1283/article_19401.shtml.

${ }^{7}$ V. V. Brazhkin, A. G. Lyapin, S. V. Popova, and R. N. Voloshin, Phys. Rev. B 51(12), 7549 (1995).

${ }^{8}$ R. J. Nelmes, M. I. McMahon, N. G. Wright, D. R. Allan, and J. S. Loveday, Phys. Rev. B 48(13), 9883 (1993).

${ }^{9}$ B. Haberl, M. Guthrie, B. D. Malone, J. S. Smith, S. V. Sinogeikin, M. L. Cohen, J. S. Williams, G. Shen, and J. E. Bradby, Phys. Rev. B 89(14), 144111 (2014).

${ }^{10}$ B. C. Johnson, B. Haberl, S. Deshmukh, B. D. Malone, M. L. Cohen, J. C. McCallum, J. S. Williams, and J. E. Bradby, Phys. Rev. Lett. 110(8), 085502 (2013).

${ }^{11}$ J. S. Williams, B. Haberl, S. Deshmukh, B. C. Johnson, B. D. Malone, M. L. Cohen, and J. E. Bradby, Phys. Status Solidi RRL 7(5), 355 (2013).

${ }^{12}$ Y. Ikoma, K. Kumano, K. Edalati, K. Saito, Q. Guo, and Z. Horita, Philos. Mag. Lett. 97(1), 27 (2017). 
${ }^{13}$ B. D. Malone, J. D. Sau, and M. L. Cohen, Phys. Rev. B 78, 035210 (2008).

${ }^{14}$ J. D. Joannopoulos and M. L. Cohen, Phys. Rev. B 7(6), 2644 (1973).

${ }^{15}$ Z. Zhao, H. Zhang, D. Y. Kim, W. Hu, E. S. Bullock, and T. A. Strobel, Nat. Commun. 8, 13909 (2017).

${ }^{16}$ M. Voros, S. Wippermann, B. Somogyi, A. Gali, D. Rocca, G. Galli, and G. T. Zimanyi, J. Mater, Chem. A 2(25), 9820 (2014).

${ }^{17}$ Y. Ikoma, K. Kumano, K. Edalati, M. R. McCartney, D. J. Smith, and Z. Horita, Mater. Charact. 132, 132 (2017).

${ }^{18}$ E. López-Cruz and M. Cardona, Solid State Commun. 45(9), 787 (1983).

${ }^{19}$ S. Deshmukh, B. Haberl, S. Ruffell, P. Munroe, J. S. Williams, and J. E. Bradby, J. Appl. Phys. 115(15), 153502 (2014).

${ }^{20}$ L. A. Giannuzzi, B. W. Kempshall, S. M. Schwarz, J. K. Lomness, B. I. Prenitzer, and F. A. Stevie, in Introduction to Focused Ion Beams: Instrumentation, Theory, Techniques and Practice, edited by L. A. Giannuzzi and F. A. Stevie (Springer US, Boston, MA, 2005), p. 201.

${ }^{21}$ B. D. Malone and M. L. Cohen, Phys. Rev. B 86(5), 054101 (2012).

${ }^{22}$ M. A. Paesler, D. E. Sayers, R. Tsu, and J. Gonzalez-Hernandez, Phys. Rev. B 28(8), 4550 (1983); G. W. Johnson, D. E. Brodie, and E. D. Crozier, Can. J. Phys. 67(4), 358 (1989).
${ }^{23}$ S. V. Demishev, D. G. Lunts, D. V. Nekhaev, N. E. Sluchanko, N. A. Samarin, V. V. Brazhkin, A. G. Lyapin, S. V. Popova, and N. N. Mel'nik, J. Exp. Theor. Phys. 82(6), 1159 (1996), available at http://www.jetp.ac. ru/cgi-bin/e/index/e/82/6/p1159?a=list.

${ }^{24}$ R. O. Piltz, J. R. Maclean, S. J. Clark, G. J. Ackland, P. D. Hatton, and J. Crain, Phys. Rev. B 52(6), 4072 (1995).

${ }^{25}$ J. S. Kasper and R. H. Wentorf, Science 197(4303), 599 (1977).

${ }^{26}$ L. Rapp, B. Haberl, C. J. Pickard, J. E. Bradby, E. G. Gamaly, J. S. Williams, and A. V. Rode, Nat. Commun. 6, 7555 (2015).

${ }^{27}$ B. C. Johnson, P. Gortmaker, and J. C. McCallum, Phys. Rev. B 77(21), 214109 (2008).

${ }^{28}$ G. L. Olson and J. A. Roth, Mater. Sci. Rep. 3(1), 1 (1988).

${ }^{29}$ R. Lieten Ruben, M. Tatsuro, J. Wipakorn, H. Hiroyuki, U. Noriyuki, M. Shu, T. Masatoshi, and L. Jean-Pierre, Appl. Phys. Express 6(10), 101301 (2013).

${ }^{30}$ Z. Pan, H. Sun, Y. Zhang, and C. Chen, Phys. Rev. Lett. 102(5), 055503 (2009).

${ }^{31}$ CRC Handbook of Chemistry and Physics, 61st ed. (CRC Press, Florida).

${ }^{32}$ B. Haberl, M. Guthrie, S. V. Sinogeikin, G. Shen, J. S. Williams, and J. E. Bradby, High Pressure Res. 35(2), 99 (2015). 